\title{
Eosinophilic Pneumonia Associated With Azacitidine in a Patient With Myelodysplastic Syndrome
}

\author{
Girish B Nair MD, Melissa Charles MD, Lorna Ogden MD, and Peter Spiegler MD
}

\begin{abstract}
Eosinophilic pneumonia is characterized by cough, lung infiltrates on imaging, and by the presence of eosinophils in the alveoli and pulmonary interstitium. Azacitidine, a pyramidine nucleoside analog of cytidine, is FDA approved for the treatment of various myelodysplastic syndromes. We present a case of a 76-year-old man with recently diagnosed myelodysplastic syndrome, who developed eosinophilic pneumonia after initiating therapy with azacitidine. There was clinical and radiographic improvement with cessation of the drug and treatment with prednisone. Diagnosis of drug-induced eosinophilic pneumonia is established by having a temporal relationship between onset of symptoms and initiation of therapy, bronchoalveolar lavage or lung biopsy evidence of pulmonary eosinophilia, no other explanation for the disease, and improvement upon cessation of the offending agent. Our case illustrates the need for a high index of suspicion to identify adverse pulmonary reactions associated with newly developed medications. Key words: azacitidine; eosinophilic pneumonia; myelodysplastic syndrome; pulmonary infiltrates. [Respir Care 2012;57(4):631-633. (C) 2012 Daedalus Enterprises]
\end{abstract}

\section{Introduction}

Eosinophilic pneumonia is characterized by cough, lung infiltrates on imaging, and by the presence of eosinophils in the alveolar spaces and pulmonary interstitium. Azacitidine, a pyramidine nucleoside analog of cytidine, is FDA approved for the treatment of various myelodysplastic syndromes. We present the case of a patient with eosinophilic pneumonia associated with azacitidine. Our case is the first reported case of azacitidine-induced eosinophilic pneumonia, to the best of our knowledge.

\section{Case Report}

A 76-year-old man with recently diagnosed myelodysplastic syndrome presented with dyspnea of 1 month's

The authors are affiliated with the Division of Pulmonary and Critical Care, Winthrop University Hospital, Mineola, New York.

The authors have disclosed no conflicts of interest.

Correspondence: Girish B Nair MD, Division of Pulmonary and Critical Care Medicine, Winthrop University Hospital, 222 Station Plaza North, Suite 400, Mineola NY 11501. E-mail: gbnair@winthrop.org.

DOI: $10.4187 /$ respcare. 01338 duration, non-productive cough, and fever for 1 week. He had a $7 \mathrm{~mm}$ pulmonary nodule, which remained stable in follow-up over a 3-year period. He completed his second treatment with azacitidine $\left(75 \mathrm{mg} / \mathrm{m}^{2}\right.$ for $7 \mathrm{~d}$, subcutaneous) 3 weeks prior to admission. Chronic home medications were losartan, esomeprazole, aspirin, folic acid, and a multivitamin. He worked as a police psychologist and had no recent travels. He was not a cigarette smoker, but smoked pipes for 3 years, 30 years in the past. He denied recreational drug use.

His temperature was $38.3^{\circ} \mathrm{C}$. Chest auscultation revealed diminished breath sounds, with bilateral rales. The leukocyte count was normal at 4,700 cells $/ \mu \mathrm{L}$ (59\% neutrophils, $5 \%$ eosinophils), and the platelet count was elevated at 617,000 cells $/ \mu \mathrm{L}$. The chest radiograph showed bilateral interstitial infiltrates, with a peripheral and basilar predominance. The computed tomography scan of the chest showed diffuse bilateral patchy pulmonary infiltrates, which were more pronounced in the peripheries, with surrounding ground-glass opacities, and enlargement of the mediastinal and bilateral hilar lymph nodes (Fig. 1). The patient was started on ceftriaxone and azithromycin intravenously. An outside computed tomography scan done 5 months ago, prior to initiation of azacitidine, demonstrated clear lung fields bilaterally, except for the stable left lower lobe lung nodule (Fig. 2). Arterial blood gas 


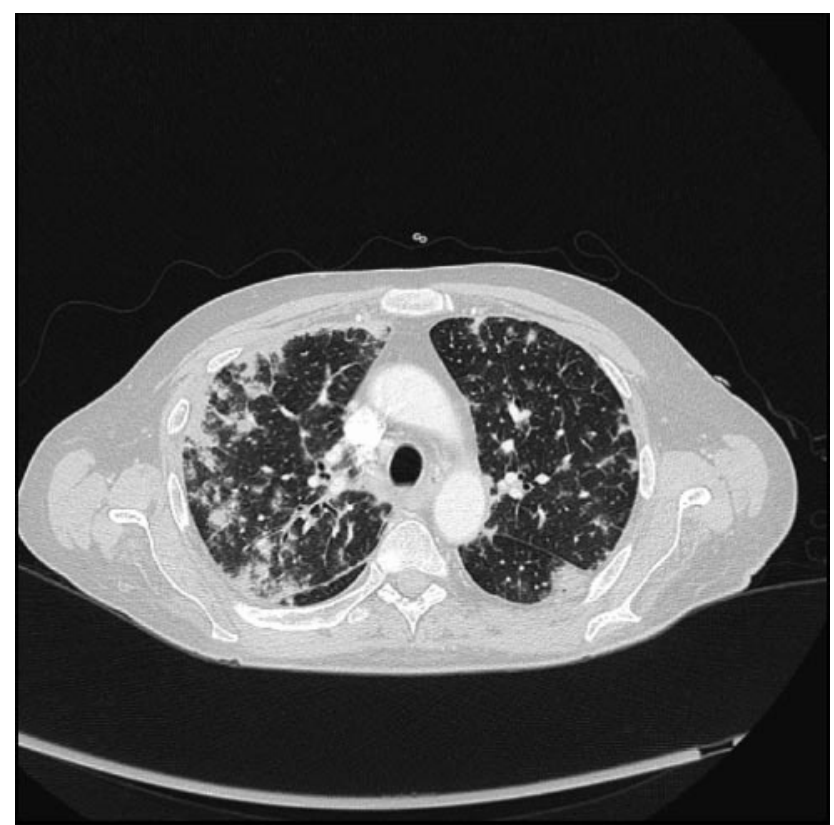

Fig. 1. A computed tomographic scan of the chest shows diffuse bilateral patchy pulmonary infiltrates predominant in the peripheries, with surrounding ground-glass opacities and enlargement of the mediastinal and bilateral hilar lymph nodes.

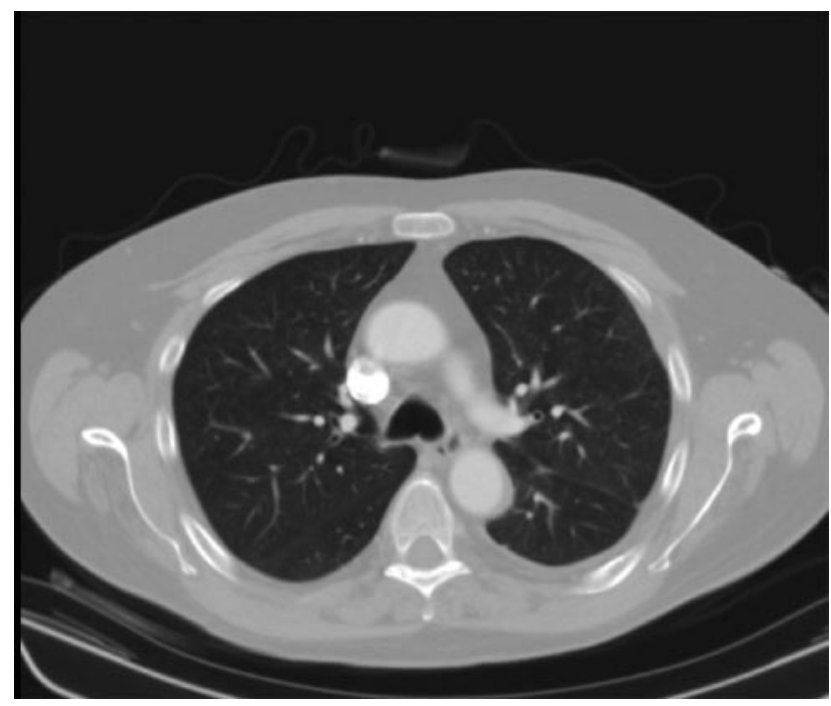

Fig. 2. A representative image from a computed tomographic scan performed 5 months prior to initiation of azacitidine demonstrates normal lung parenchyma.

analysis showed a $\mathrm{pH}$ of $7.47, \mathrm{P}_{\mathrm{aCO}}$ of $33 \mathrm{~mm} \mathrm{Hg}$, and $\mathrm{P}_{\mathrm{aO}}$ of $61 \mathrm{~mm} \mathrm{Hg}$ on room air. Additional tests for antinuclear antibody and anti-neutrophil cytoplasmic antibody were performed and were within normal limits. An immunoglobulin-E level was elevated at 10,954 IU/mL (normal $<200 \mathrm{IU} / \mathrm{mL}$ ).

Bronchoscopy was performed. The transbronchial lung biopsy showed organizing pneumonia with intra-alveolar

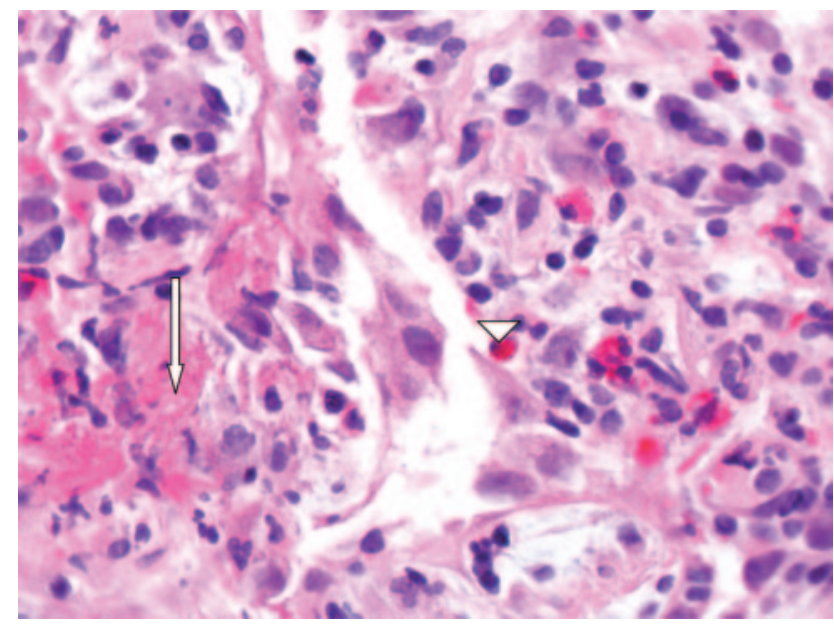

Fig. 3. Lung biopsy on high power microscopy $(400 \times)$ showing fibrin exudate (arrow) and increased eosinophils (arrow head), along with lymphocytes and fibroblasts in the intra-alveolar spaces and septae.

plugs of fibroblastic tissue, with predominant eosinophilic infiltration, consistent with eosinophilic pneumonia (Fig. 3). Fungal stains on pathology were negative. Blood and bronchoscopic cultures were negative. Azacitidine and antibiotic therapy was discontinued, and the patient was started on intravenous solumedrol, $1 \mathrm{mg} / \mathrm{kg}$ twice daily. The patient had symptomatic improvement and was discharged on prednisone on the 6th hospital day. Prednisone was tapered over the following 2 months, and there was complete radiographic clearing.

\section{Discussion}

Drug-induced eosinophilic pneumonia can be caused by several medications. Symptoms typically develop within 8 weeks of drug initiation, but may be delayed. Patients usually present with cough, wheeze, and slowly progressive dyspnea. Computed tomography findings in drug-induced eosinophilic lung disease usually show bilateral airspace consolidation and ground-glass opacities in a peripheral distribution, often involving the middle and upper lung zones. ${ }^{1}$ Histological findings include diffuse alveolar damage, nonspecific interstitial pneumonia, and organizing pneumonia. ${ }^{2,3}$ Diagnosis is often delayed, since many other diseases can have a similar presentation (Table 1). If not identified early, it can progress to respiratory failure.

Azacitidine acts by causing DNA hypo-methylation, restoring normal tumor suppressor gene function, and control of cellular differentiation and proliferation. The major adverse effects reported with use of azacitidine include myelo-suppression, hepatic coma, renal tubular acidosis, bone aches, and convulsions. The reported pulmonary tox- 


\section{Eosinophilic Pneumonia Associated With Azacitidine}

Table 1. Select List of Differential Diagnosis of Pulmonary Eosinophilia

Medications: including over-the-counter and herbal remedies Infections: parasitic, fungal infections

Vasculitides: Churg-Strauss syndrome, Wegener granulomatosis Myeloproliferative diseases: hyper-eosinophilic syndrome Idiopathic causes: acute and chronic eosinophilic pneumonia Other conditions: asthma, sarcoidosis, organizing pneumonia

icities are mainly acute fibrinous and organizing pneumonia, lung infiltration, interstitial and alveolar fibrosis, and hemoptysis. ${ }^{4,5}$ The mechanism of injury is thought to be related to activation of cytokines such as interleukin-5 from helper $\mathrm{T}$ cells sensitized by the drug, which in turn causes eosinophil accumulation in the lung tissue. ${ }^{2,6}$

A thorough history, including recent travel and exposure to chemicals or toxins, is the first step in establishing the diagnosis. ${ }^{2}$ The evaluation should focus on identifying other causes, such as systemic vasculitis. Bronchoalveolar lavage typically shows a high percentage of eosinophils (up to 25\%); however, it is not necessary to make the diagnosis. ${ }^{7}$ Diagnosis is established by having a temporal relation between disease onset and initiation of the offending agent, bronchoalveolar lavage or lung biopsy demonstrating eosinophilia, no other explanation for the disease, and improvement upon cessation of the offending agent.

Idiopathic eosinophilic lung disease has a similar clinical presentation and features on pathologic examination. It usually has a rapid onset, and can often progress to respiratory failure, but is quite steroid-responsive. Radio- graphic findings are often nonspecific, with bilateral ground-glass or reticular opacities. It is a diagnosis of exclusion, and, unlike our patient reported, there would be no association with a specific medication. Chronic eosinophilic pneumonia often has an insidious onset, with marked peripheral eosinophilia, and, while responsive to steroid therapy, recurrences are common. ${ }^{7}$ The chest radiograph classically shows bilateral, peripheral infiltrates, the "photograph-negative" of pulmonary edema. Our case illustrates the need for a high index of suspicion to identify adverse pulmonary reactions associated with novel medications.

\section{REFERENCES}

1. Souza CA, Müller NL, Johkoh T, Akira M. Drug-induced eosinophilic pneumonia: high-resolution CT findings in 14 patients. AJR Am J Roentgenol 2006;186(2):368-373.

2. Allen JN. Drug-induced eosinophilic lung disease. Clin Chest Med 2004;25(3):77-88.

3. Rossi SE, Erasmus JJ, McAdams HP, Sporn TA, Goodman PC. Pulmonary drug toxicity: radiologic and pathologic manifestations. Radiographics 2000;20(5):1245-1259.

4. Adams CD, Szumita PM, Baroletti SA, Lilly CM. Azacitidine-induced interstitial and alveolar fibrosis in a patient with myelodysplastic syndrome. Pharmacotherapy 2005;25(5):765-768.

5. Vasu TS, Cavallazzi R, Hirani A, Marik PE. A 64-year-old male with fever and persistent lung infiltrate. Respir Care 2009;54(9): 1263-1265.

6. Kita H, Sur S, Hunt LW, Edell ES, Weiler DA, Swanson MC, et al. Cytokine production at the site of disease in chronic eosinophilic pneumonitis. Am J Respir Crit Care Med 1996;153(4):1437-1441.

7. Li H, Groshong SD, Lynch D, Brown KK, Frankel SK. Eosinophilic lung disease. Clin Pulm Med 2010;17(2):66-74. 